\title{
LINE-1 in cancer: multifaceted functions and potential clinical implications
}

\author{
Lu Xiao-Jie, MD1', Xue Hui-Ying2 ${ }^{2}$ Xiaolong Qi, MD, ${ }^{3,4}$ Xu Jiang ${ }^{5}$ and Ma Shi-Jie ${ }^{6}$
}

\begin{abstract}
Long interspersed nuclear element-1 (L1) retrotransposons are jumping genes that comprise $17 \%$ of human DNA. They utilize a "copy-and-paste" mechanism to propagate themselves throughout the genome via RNA intermediates, a process termed retrotransposition. L1s are active in the germ line and during embryogenesis, yet they are epigenetically suppressed in somatic cells. In cancer cells, however, L1s are aberrantly activated and may have a role in genome instability, one of the hallmarks of cancer pathogenesis. Their methylation states and retrotransposition activities are associated with and fluctuate during cancer initiation and progression, thus representing promising diagnostic biomarkers and therapeutic targets. During tumorigenesis, L1s exert both retrotransposition-dependent and retrotransposition-independent functions. The former may result in alterations in target gene expression or chromosomal rearrangement, or drive Alu and SVA, events that could function in tumorigenesis,
\end{abstract}

whereas the latter can potentially exert epigenetic regulation by generating endo-siRNAs, forming chimeric L1 transcripts or changing the expression of adjacent genes by providing novel splicing sites or alternative promoters. Moreover, the L1 encoded proteins, ORF1p and ORF2p, may have pro-oncogenic potential by, for example, activating oncogenic transcriptional factors or sequestering oncosuppressors. Herein, we introduce the components and mechanisms of L1 retrotransposition, discuss the landscape, possible functions, and regulation of L1 activity in cancer, and seek their potential as diagnostic biomarkers and therapeutic targets.

Genet Med advance online publication 3 September 2015

Key Words: biomarker; cancer; insertional mutagenesis; LINE-1; retrotransposition
The human genome is abundant with interspersed repetitive sequences originated from retrotransposons. Until now, three categories of retrotransposons have remained unequivocally active: LINE-1(L1), Alu, and SVA elements. The first one is autonomous-capable of self-propagation through RNA intermediates - and the latter two are nonautonomous and thus rely on L1 for mobilization. There are approximately 500,000 L1 copies in the human genome, composing $17 \%$ of human DNA. ${ }^{1}$ However, the majority of L1s have lost retrotransposition competency due to $5^{\prime}$ truncations, inverted rearrangements, or point mutations occurring during reverse transcription or subsequent chromosomal replication of the inserted element. It is estimated that the average human genome contains $\sim 50-120$ active L1s, with a highly active subset $(\sim 5-10 \%$ of active elements) termed hot L1s comprising the majority of this activity. ${ }^{2}$ These active L1s utilize a "copy-and-paste" mechanism to insert themselves throughout the genome, with potentially disruptive effects on neighboring genes or regulatory sequences. In this way, active L1s keep reshaping the human genome and become a source of endogenous mutagenesis that causes individual genome variation and can participate in the pathogenesis of many genetic diseases, including cancer. ${ }^{3-5}$ Cancer is a genetic disease resulting from accumulated genetic mutations, to which L1 can be one contributor. In this review, we discuss the putative multilayered functions of L1s in cancer and their potential for clinical implications, with a focus on recent advances.

\section{STRUCTURE AND RETROTRANSPOSITION PROCESS OF L1}

A retrotransposition-competent human L1 is $\sim 6 \mathrm{~kb}$ in length and comprises a $5^{\prime}$ untranslated region (UTR), two open reading frames (ORF1 and ORF2), and a 3' UTR ending with a poly(A) tail (Figure 1a). ${ }^{6}$ Its $5^{\prime}$ UTR harbors two internal promoters, one is sense ${ }^{7}$ and the other is antisense ${ }^{8}$ (Figure 1a). The sense promoter binds RNA polymerase II and initiates L1 transcription from the $5^{\prime}$ end to $3^{\prime}$ end, whereas the antisense promoter can give rise to chimeric RNAs transcribed partially from L1 $5^{\prime}$ UTR and partially from neighboring sequences (also called flanking sequences).$^{7-9}$ ORF1 encodes a $40 \mathrm{kDa}$ protein (ORF1p) harboring a RNA recognition motif, whereas ORF2 encodes a $150 \mathrm{kDa}$ protein (ORF2p) with endonuclease and reverse-transcriptase (RT) activities (Figure 1a). After being transcribed into full-length mRNAs in the nucleus from the sense promoter, L1 mRNAs are transported to the cytoplasm, wherein they are

The first two authors and last two authors contributed equally to this work and the first two authors are co-first authors.

${ }^{1}$ Department of Gastroenterology, Shanghai East Hospital, Tongji University, School of Medicine, Shanghai, China; ${ }^{2}$ The Reproductive Center, Jiangsu Huai'an Maternity and Children Hospital, Huai'an, China; ${ }^{3}$ Department of General Surgery, Nanfang Hospital, Southern Medical University, Guangzhou, China; ${ }^{4}$ Department of Gastroenterology and Hepatology, Tongji Hospital, Tongji University School of Medicine, Shanghai, China; ${ }^{5}$ Department of Rehabilitation, Affiliated Huai'an Hospital of Xuzhou Medical College and Second People's Hospital of Huai'an, Huai'an, China; ${ }^{6}$ Department of Gastroenterology, Huai’an First People's Hospital, Nanjing Medical University, Huai’an, Jiangsu, China. Correspondence: Xu Jiang (luzg88@163.com) or Ma Shi-Jie (luzg1981@163.com) 
a
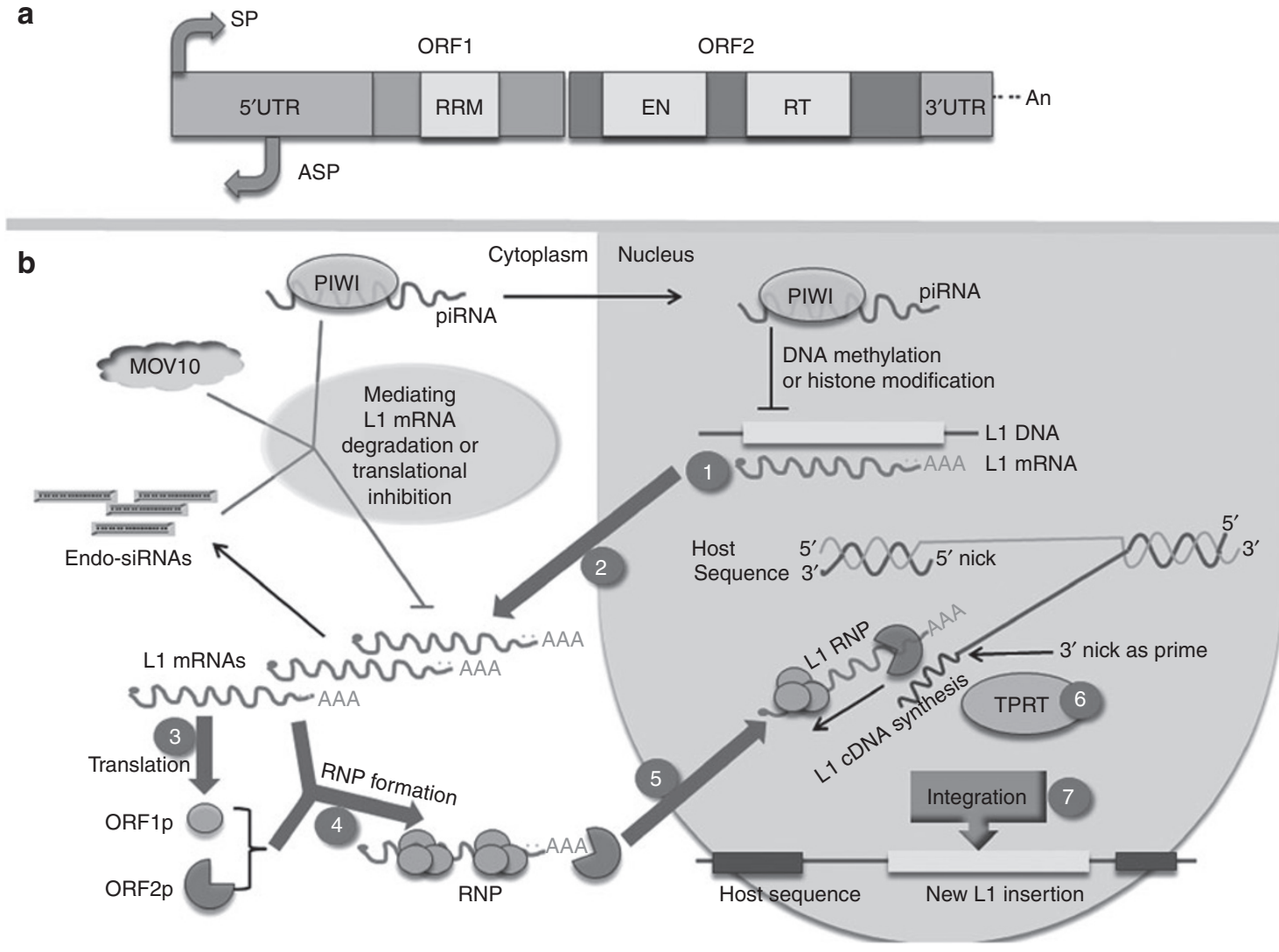

Figure 1 Schematic of a full-length L1 and the process of retrotransposition. (a) A full-length L1 comprises a 5' UTR, two open reading frames (ORF1 and ORF2), and a 3' UTR ending with a poly(A) tail. Its 5' UTR harbors two internal promoters: one is sense and the other is antisense. ORF1p, the protein encoded by ORF1, contains a RNA recognition motif, whereas ORF2p has endonuclease (EN) and reverse-transcriptase (RT) activities. (b) L1 mRNAs transcribed from the sense promoter (step 1) are transported to the cytoplasm (step 2), wherein they are suppressed or degraded by siRNAs or piRNA-mediated mechanisms, or translated into ORF1p and ORF2p (step 3), which bind to L1 mRNA to form ribonucleoprotein (RNP) (step 4). Then, the L1 RNP returns to the nucleus (step 5), wherein L1 mRNA is reverse-transcribed into CDNA (step 6) and integrated into new genomic loci (step 6) by an EN-mediated mechanism termed target site-primed reverse transcription (TPRT). During TPRT, ORF2p EN generates nicks in genomic DNA to expose 3'-OH ends that serve as primers to synthesize L1 cDNAs by ORF2p RT. ASP, antisense promoter; endo-siRNA, endogenous siRNA; L1, long interspersed nuclear element-1; piRNA, piwi-interacting RNA; siRNAs, small interfering RNAs; SP, sense promoter; UTR, untranslated region. Steps are numbered in small circles.

suppressed or degraded by small interfering RNAs (siRNAs) or piwi-interacting RNA (piRNA)-mediated mechanisms (discussed later), or translated into ORF1p and ORF2p. Both ORF1p and ORF2p preferentially bind their encoding RNA (a phenomenon called cis preference ${ }^{10}$ ) and are indispensable for L1 mobilization (Figure 1b). ${ }^{6,11}$ The resultant L1 ribonucleoprotein particles return to the nucleus where the L1 mRNA is reverse-transcribed into cDNA at the integration site via a poorly understood mechanism. This endonuclease-mediated process is termed target site-primed reverse-transcription (TPRT); ${ }^{12}$ TPRT can also occur in an endonuclease-independent fashion, where L1s integrate into preexisting DNA lesions. ${ }^{13}$ During TPRT, ORF2p endonuclease generates nicks in genomic DNA to expose $3^{\prime}-\mathrm{OH}$ ends, which serve as primers to synthesize L1 cDNAs by ORF2p RT (Figure 1b). ${ }^{11}$

Despite the cis preference of ORF1p and ORF2p for L1 mRNA, the $\mathrm{L} 1$ retrotransposition machinery can also reverse-transcribe other RNAs such as Alu RNAs $s^{14-16}$ and SVA RNAs, ${ }^{15,16}$ as well as some protein-coding mRNAs, ${ }^{17}$ thus inducing mutagenesis and contributing to human genome evolution and diversity.
As a result of the involvement of L1-induced mutagenesis in the pathogenesis of some kinds of diseases, including cancer, ${ }^{4,5}$ eukaryotic cells have developed several mechanisms to counteract L1 mobilization. Among them are the aforementioned siRNAs or piRNA-mediated mechanisms (Figure 1b). The bidirectional promoters within the L1 $5^{\prime}$ UTR can give rise to sense and antisense RNAs that could bind with each other to form double-stranded RNAs (dsRNAs). These dsRNAs are subsequently sliced by Dicer, a ribonuclease, into smaller fragments termed endogenous (endo)-siRNAs. ${ }^{18}$ The resultant endo-siRNAs can, in turn, degrade L1 mRNAs and suppress L1 retrotransposition by triggering the RNA interference mechanism, hence constituting a negative regulatory loop (Figure 1b). ${ }^{19}$ Like siRNA, piRNA can also exert a negative regulation on L1 retrotransposition. ${ }^{20}$ piRNA is a kind of single-strand small noncoding RNA transcribed from genomic loci containing repetitive elements that binds to Piwi proteins to form a complex that suppresses L1 proliferation via RNA degradation, ${ }^{21}$ DNA methylation, ${ }^{22}$ or histone modification. ${ }^{23}$ Apart from siRNA and piRNA, other defensive strategies 
against L1 mobilization include the RNA helicase MOV10 (which degrades L1 mRNAs or suppresses their translation) ${ }^{24}$ and APOBEC3 family members (Figure 1b). ${ }^{25}$ Different members of the APOBEC3 family may inhibit L1 retrotransposition by different mechanisms. ${ }^{26}$ For example, APOBEC3A inhibits L1 retrotransposition by mutating $\mathrm{L} 1$ cDNA during TPRT, ${ }^{27}$ whereas APOBEC3C inhibits L1 retrotransposition by interaction with ORF1p. ${ }^{28}$

\section{LANDSCAPE OF L1 RETROTRANSPOSITION IN CANCER}

L1s are active in the germ line and during embryogenesis, ${ }^{29,30}$ yet they are epigenetically suppressed in somatic cells. ${ }^{22,25}$ However, in line with L1 hypomethylation during tumorigenesis, ${ }^{31-34} \mathrm{~L} 1 \mathrm{~s}$ can be reactivated and may participate in the pathological processes of cancer initiation and progression. ${ }^{3,9,35}$ In 1988, Morse et al. ${ }^{36}$ reported a case of $\mathrm{L} 1$ insertion into $c$-myc gene in a breast cancer sample. In 1992, cancer-associated L1 mutagenesis was reported when somatic L1 insertion into the APC gene was found to cause gene disruption in a colon cancer sample. ${ }^{3}$

L1 activity differs among and within cancer types ${ }^{32,35}$ and fluctuates during cancer evolution. ${ }^{32,33,37}$ In a study by Lee et al., ${ }^{35}$ for example, somatic L1 insertions are more frequently found in colorectal cancer (CRC) than in prostate and ovarian cancers, whereas in multiple myeloma and glioblastoma, no somatic L1 insertions were detected. Among cancer tissues obtained from different CRC patients, the numbers of somatic L1 insertions range from 2 to 106, indicating the potential of the L1 retrotransposition profile as a signature for cancer subtyping. In African Americans with CRC, L1s were found to be progressively hypomethylated in the normal adenoma cancer sequence. ${ }^{32}$ In CRC with liver metastasis, L1 methylation level is lower in metastasis versus primary CRC tissue. ${ }^{38}$ These two examples indicate that L1 expression can change with tumor progression (also discussed below in "Functions of L1-Encoded Proteins in Cancer"). Target-site analysis revealed that somatic L1 insertions are biased away from transcriptional active regions ${ }^{35}$ and toward regions such as intergenic or heterochromatic regions, ${ }^{37}$ cancer-specific hypomethylation regions, ${ }^{35}$ or genes frequently mutated in cancer, suggesting a possible oncogenic role of L1 insertions given that frequently mutated genes are candidate drivers of tumorigenesis. ${ }^{39}$ The majority of inserted L1s are truncated rather than full-length, and thus lose the competency of further retrotransposition. ${ }^{35}$ In rare cases, however, inserted L1s are full-length and capable of retrotransposing consecutively. ${ }^{37}$

Many studies have investigated the associations between L1 methylation levels (or L1 activity) and cancer risk, progression, and prognosis, with a majority of them supporting correlations between tissue L1 hypomethylation and increased cancer risk or poor prognosis. ${ }^{32,33,40-42}$ In people with CRC family history, for example, colonic L1 hypomethylation confers a higher CRC risk. ${ }^{42}$ Another study ${ }^{43}$ revealed that L1 hypomethylation in normal colon tissue predicts predisposition to multiple colonic tumors. In cervical carcinoma samples, L1 hypomethylation levels were found to be significantly higher than those of paracancerous tissues. ${ }^{44} \mathrm{~L} 1$ hypomethylation has been reported to correlate with unfavorable prognosis of many cancers such as CRC,${ }^{45}$ hepatocellular carcinoma (HCC),${ }^{46}$ gastric cancer ${ }^{47}$ and esophageal cancer. ${ }^{34}$ The result of a meta-analysis ${ }^{48}$ also supports the correlation between L1 hypomethylation and poor prognosis of cancer patients.

However, controversies exist regarding L1 methylation status in peripheral blood of cancer patients and its prognostic implications. Compared with normal controls, L1 hypermethylation was observed in white blood cells of pancreatic, ${ }^{49}$ epithelial ovarian, ${ }^{50}$ and colorectal ${ }^{51}$ cancer patients. As for melanoma ${ }^{52}$ and $\mathrm{HCC},{ }^{53}$ serum L1s were hypomethylated relative to normal controls. In a study comparing breast cancer patients and their unaffected sisters, no association between breast cancer and L1 methylation status of white blood cells was observed. ${ }^{54}$ Whereas in a prospective study, women with lower L1 methylation levels in peripheral blood (whole blood samples were tested) were found to have an increased breast cancer risk. ${ }^{41}$ Regarding gastric cancer, studies ${ }^{55,56}$ failed to observe a correlation between white blood cell L1 methylation status and cancer prognosis. These discrepancies may be rooted in differences in cancer type, study design, and blood components tested (whole blood, serum, or white blood cells). Further research is required to determine whether increases in L1 expression present in cancer are an effect of global hypomethylation or a cause of carcinogenesis. Indeed, further studies must also determine how L1 expression results in additional retrotransposition, and how correlated the increase in L1 expression and retrotransposition are with the pathogenesis of different types of cancer and their metastases.

\section{FUNCTIONS OF L1S IN CANCER Retrotransposition-dependent functions}

Target gene (in)activation. L1 insertions may alter targetgene expression levels, ${ }^{35,57}$ which are influenced by cell type ${ }^{44}$ and the orientations of L1 insertion. ${ }^{35,58}$ It is reported that sense insertions are more likely to be disruptive, ${ }^{35}$ possibly due to the fact that L1 sense strand contains more cryptic polyadenylation sites than antisense strand. ${ }^{57}$ Generally speaking, L1 insertions are more likely to suppress than to activate target-gene expression. ${ }^{35} \mathrm{~L} 1$ insertions can contribute to tumorigenesis by inactivating tumor suppressor genes (Figure 2a) or activating oncogenes (Figure 2b).

A study on $\mathrm{L} 1$ retrotransposition in $\mathrm{HCC}^{59}$ for example, found that germ line L1 insertions into tumor suppressor gene $M C C$ can suppress MCC expression and result in elevated $\beta$-catenin protein level (MCC is an upstream inhibitor of the Wnt/ $\beta$-catenin pathway in HCC). Given the oncogenic role of the Wnt/ $\beta$-catenin pathway in HCC, this study ${ }^{59}$ suggests the possible involvement of L1 insertions in HCC predisposing mutations. This study ${ }^{59}$ also observed a gene activation event induced by cancer-specific L1 insertion. This insertion increased the expression level of ST18 (suppression of tumorigenicity 18) gene that encodes a zinc-finger transcription factor, 
a

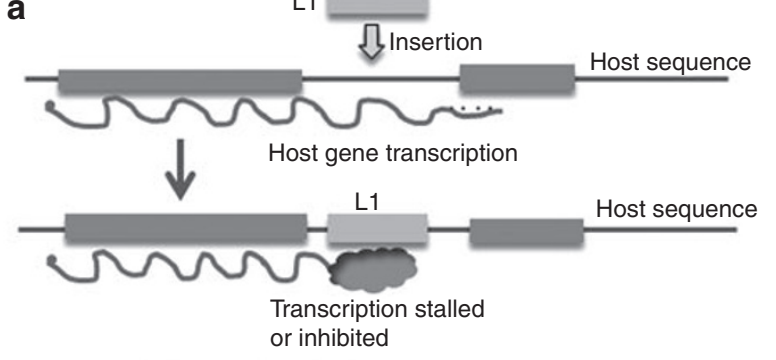

b

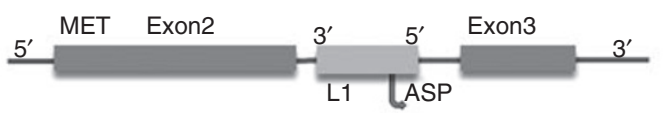

Transcription

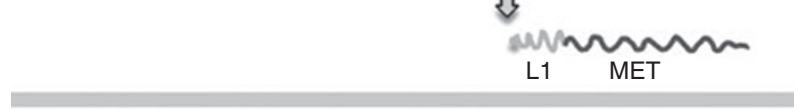

c

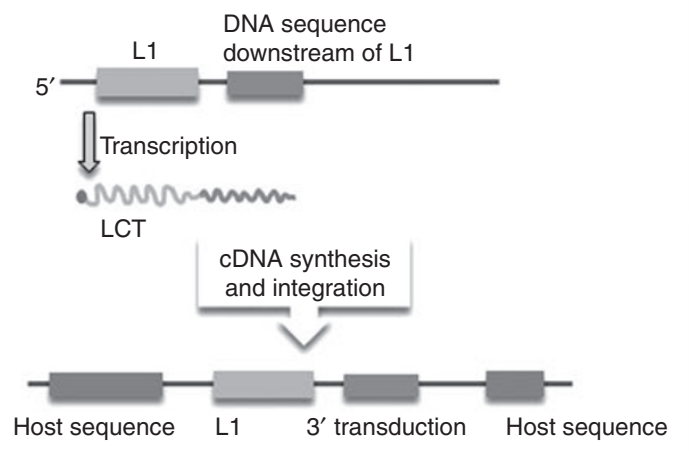

d

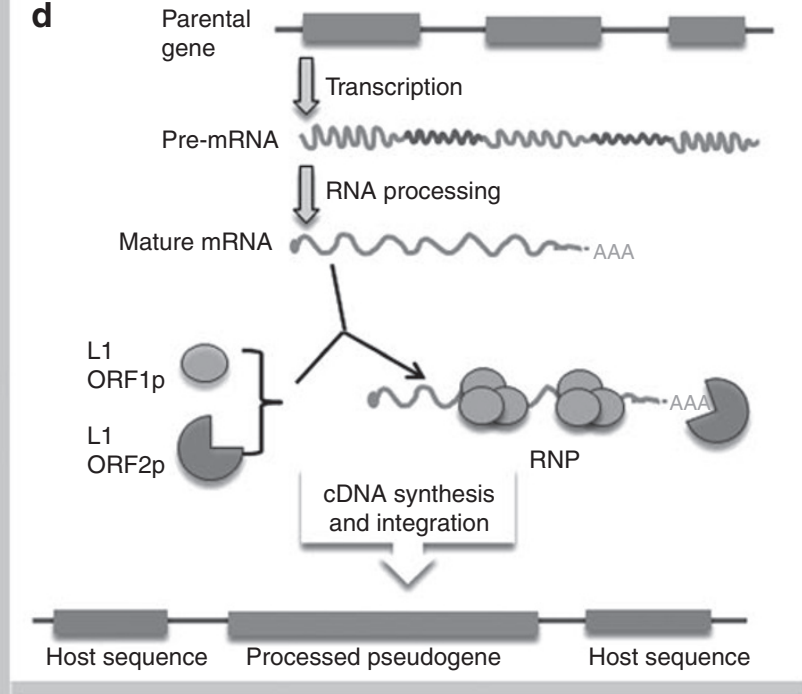

e
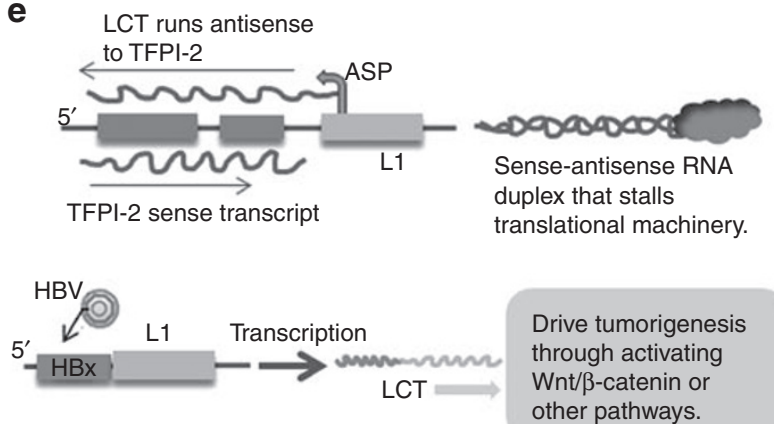

Drive tumorigenesis through activating Wnt//-catenin or other pathways.

Figure 2 Representation of potential pathogenic functions of L1 in cancer. (a) L1 insertion-mediated inhibition of host gene transcription: L1 can potentially act to slow RNA pol II elongation, dissociate it from the template, or induce premature termination of transcription. (b) L1 insertion-mediated oncogene activation: the ASP within L1 inserted antisense to gene MET serves as a transcription start site to drive MET expression. (c) 3' transduction: downstream sequence of $\mathrm{L}^{1} \mathrm{3}^{\prime}$ end is transcribed together with $\mathrm{L} 1$ and the resultant $L C T$ is reverse-transcribed and integrated into a new locus by $\mathrm{L} 1$ retrotransposition machinery. (d) L1-mediated formation of processed pseudogenes: mature mRNA (lacking introns) is reverse-transcribed and integrated into a new locus by the L1 retrotransposition machinery to generate processed pseudogenes that lack introns and are punctuated by a $3^{\prime}$ poly-A tail. (e) Functions of LCTs in cancer: ASP within L1 drives the transcription of LCT that runs antisense to upstream TFPI-2 gene. The expression of TFPI- 2 is inhibited by this LCT (upper). HBx from the HBV genome drives LCT that is transcribed partially from HBx and partially from L1 sequence. This LCT functions as an oncogenic long noncoding RNA that can activate Wnt/ $\beta$-catenin pathway (bottom). For simplicity, only two exons are shown for gene MET and TFPI-2. ASP, antisense promoter; HBV, hepatitis B virus; L1, long interspersed nuclear element-1; LCT, L1 chimeric transcript; ORF1p, protein encoded by L1 open reading frame 1; ORF2p, protein encoded by L1 open reading frame 2; RNP, ribonucleoprotein.

possibly through severing one of its enhancers that is otherwise bound by ST18 protein to exert negative self-regulation.

The promoters within L1 can provide an alternative start site for transcription of neighboring oncogenes (Figure $2 \mathbf{b}$ ), which is exemplified by L1 insertion-mediated gene activation of oncogene Met. In CRC and HCC, L1 insertion into the intronic region of MET (L1-MET) leads to elevated expression of MET. ${ }^{38,46}$ In CRC and associated liver metastasis tissue, the hypomethylation of L1 ASP within L1-MET is correlated with elevated MET expression. ${ }^{38}$ In HCC, L1-MET was also identified and its hypomethylation was found to be correlated with elevated $c$-MET expression. ${ }^{46}$

Chromosomal rearrangements and processed pseudogenes. L1 insertions are capable of generating various forms of chromosomal rearrangements, such as genomic deletions, ${ }^{60}$ duplications, ${ }^{61}$ inversions, ${ }^{61}$ or translocations. ${ }^{37}$ During tumorigenesis, chromosomal rearrangements can give rise to oncogene duplications/activations, tumor suppressor deletions, or oncogenic fusion proteins. Given the importance of chromosomal rearrangements in cancer pathogenesis and the frequency of L1-induced chromosomal rearrangements, they constitute a potential contributor to tumorigenesis.

Han et al., ${ }^{60}$ for example, identified 24 L1-mediated deletions in human genome since our divergence from our common ancestor with chimpanzees. In HeLa cells, artificially induced L1 retrotransposition gave rise to not only sequence deletions but also duplications and inversions. ${ }^{61}$ When mature mRNAs are retrotranscribed and integrated into new loci by L1 retrotransposition machinery, they become processed 
pseudogenes (Figure 2d). Pseudogenes were once regarded as nonfunctional "junk DNA." Recently, however, emerging evidence has suggested that some of them might play multifaceted roles at DNA, RNA, or protein levels during tumorigenesis. ${ }^{62}$ Therefore, L1-mediated pseudogene formations represent another layer of functionality of L1s in cancer. Cooke et al., ${ }^{20}$ for example, studied somatic pseudogene profiles across 18 tumor types and reported that a substantial part of somatically acquired pseudogenes are generated by L1 retrotransposition during cancer development. Among the tumor types studied, ${ }^{20}$ non-small cell lung cancer and CRC are two cancers in which somatic pseudogenes are most frequently identified, in line with previous reports ${ }^{35,37}$ that L1s are active in these two cancer types.

3' Transduction. In some cases, sequence downstream of the $3^{\prime}$ end of L1s may be transcribed along with L1s and concomitantly retrotransposed into target sites, a process termed $3^{\prime}$ transduction (Figure 2c).

In a recent study ${ }^{37}$ profiling $\mathrm{L} 1$ retrotransposition events in 290 cancer samples from 244 patients across 12 tumor types, $24 \%$ of these events were accompanied by $3^{\prime}$ transductions, with half of them being orphan ones in which downstream sequences alone (without any accompanying L1 component) were mobilized by L1 retrotransposition machinery. The size of a transduced sequence ranges from less than $0.2 \mathrm{~kb}$ to approximately $12 \mathrm{~kb}$, with the majority of them being less than $1 \mathrm{~kb}$. In certain cancer types such as lung cancer, $3^{\prime}$ transduction represents a considerable form of genomic alterations. ${ }^{37}$ These $3^{\prime}$ transductions can be regarded as a very small duplication of a segment of DNA, and therefore they have the potential to shuffle exons of genes. ${ }^{63}$ The functional consequences of these $3^{\prime}$ transductions in cancer are interesting areas for further investigation.

Besides the aforementioned mechanisms, L1 can also mobilize other nonautonomous retrotransposons such as Alu and SVA, ${ }^{14-16}$ potentially leading to additional genomic lesions that could function in tumorigenesis. We do not focus on this topic due to space limitations and the availability of recent reviews on Alu and SVA transposition. ${ }^{14,15}$

\section{Retrotransposition-independent functions.}

In addition to retrotransposition-dependent functions, L1s are capable of exerting many retrotransposition-independent impacts on gene expression through L1-derived regulatory RNAs, L1 chimeric transcripts (LCTs), or L1-mediated transcriptional interference. An excellent example of this comes from the participation of $\mathrm{L} 1$ in $\mathrm{X}$ chromosome inactivation during embryo development. ${ }^{64}$ In a HepG2 cell line, ${ }^{65}$ ectopic L1 expression resulted in detectable expression changes of 24 genes, with half of them being retrotransposition-independent.

L1-derived regulatory RNAs. As stated, dsRNAs derived from L1 sense and antisense RNAs can be processed by Dicer into endo-siRNAs that trigger the RNA interference mechanism and exert extensive epigenetic regulation. ${ }^{19}$ Besides endo-
siRNAs, a small part of miRNAs and miRNA response elements in the $3^{\prime}$ UTRs of target genes were also reported to derive from L1s. ${ }^{66}$ In addition, evidence ${ }^{67}$ showed that L1-derived sequences exist within or nearby transcription start sites of many long noncoding (lnc)RNAs and participate in lncRNA expression and processing. miRNAs and lncRNAs may regulate gene expression directly or indirectly through competing endogenous RNA (ceRNA) networks. ${ }^{68}$ In normal somatic cells, L1 retrotransposition is suppressed ${ }^{22,31}$ and L1 transcripts are degraded or processed into regulatory RNAs that maintain cell homeostasis, ${ }^{19,21,24,66,67}$ whereas in cancer, one study found that L1 transcripts were potentially biased toward cDNA formation and retrotransposition, ${ }^{69}$ leading to speculation that deregulated regulatory RNA networks may drive tumorigenesis.

L1 chimeric transcripts. In addition to these regulatory RNAs, L1s can regulate gene expression through LCTs, which are driven by promoters within ${ }^{9}$ or outside ${ }^{69} \mathrm{~L} 1$ sequences. A large LCT detected in breast and colon cancers, for example, is driven by the $\mathrm{L} 1$ antisense promoter and includes a portion of the TFPI-2 gene, a metastasis suppressor. ${ }^{9}$ This LCT includes TFPI2 antisense RNA, which can lead to epigenetic silence of TFPI2 in a transgenic mouse embryonic stem cell model, ${ }^{9}$ possibly through sense-antisense duplex formation that stalls TFPI-2 mRNA translation (Figure 2e, upper). In human breast and colon cancer cell lines, the expression of this LCT was found to be associated with decreased TFPI-2 expression. ${ }^{9}$

In hepatitis B virus-positive HCC tissues, Lau et al..$^{70}$ detected a 674 bp chimeric $\mathrm{HBx}-\mathrm{L} 1$ transcript that is driven by viral $H B x$ promoter and correlates with unfavorable prognosis of patients. Subsequent functional investigations revealed that $\mathrm{HBx}-\mathrm{L} 1$ functions as an lncRNA to promote cell mobility in HCC cells through epithelial-to-mesenchymal transition and to promote chemical-induced hepatocarcinogenesis in a mouse model via activation of the Wnt/ $\beta$-catenin pathway (Figure 2e, bottom).

L1-mediated transcriptional interference. L1 sequences can interfere with host gene transcription in many ways. They may be able to, for example, slow RNA pol II elongation, dissociate it from the template, or induce premature termination of transcription. ${ }^{71}$ They can also mediate transcriptional interference via alternative splicing. Different splicing variants (SVs) of the same gene may play different roles in physiological conditions as well as in cancer. An example comes from transcript factor KLF6 and its SVs. Wild-type KLF6 is generally regarded as an oncosuppressor, whereas KLF6 SV1 plays an oncogenic role in many cancers. ${ }^{72}$ During tumorigenesis, RNA splicing may bias toward oncogenic SVs to support cancer initiation and progression. ${ }^{73}$ Alternative splicing occurs in $95 \%$ of multi-exon genes ${ }^{73}$ and, in a small minority of cases, may be influenced by L1, resulting in transcriptional interference. The L1 sequence contains polyadenylation sites as well as donor and acceptor splice sites that may induce novel alternative splicing via retention, exonization, or polyadenylation of the upstream intronic sequences. ${ }^{57}$ These L1-induced SVs may occasionally 
generate novel protein isoforms with new functions, which may serve as a mutation reservoir for tumor evolution.

\section{FUNCTIONS OF L1-ENCODED PROTEINS IN CANCER}

Because L1 expression is apparently activated (or upregulated) in some cancers, the L1-encoded proteins are also detectable. ${ }^{31}$ They may participate in tumorigenesis and their expression profiles can be of potential clinical significance. A study of breast cancer by Chen et al. ${ }^{74}$ for example, revealed that the majority of invasive cancers expressed L1 proteins in the cytoplasm, with $28-31 \%$ of them showing nuclear expression. Moreover, patients with L1 nuclear expression suffered from more lymph node metastasis and worse prognosis relative to patients without. Given that nuclear ORF1p and ORF2p are building blocks for L1 retrotransposition, whether or not this prognostic association is attributable to retrotransposition-induced mutagenesis requires further investigation.

\section{ORF1p}

ORF1p is a $40 \mathrm{kDa}$ protein with RNA-binding capacity. In a study encompassing 1,027 cancer samples across more than 20 cancer types, ORF1p were detectable in $47 \%$ of all cancer samples, especially in highly malignant samples, but rarely in earlystage cancers and absent from normal somatic tissues. ${ }^{31}$ Among different cancer types, the frequencies of detectable ORF1p are different. This common yet cancer-specific expression profile of ORF1p warrants further investigation regarding whether it can be used to assist in cancer diagnosis in the future.

In an HCC cell line and xenograft mouse model, ${ }^{75}$ overexpression and knockdown (by RNA interference) of ORF1p led to proproliferative and antiproliferative effects, respectively, suggesting an oncogenic role. Subsequent exploration of the mechanism revealed that ORF1p sequestrates cytoplasmic SMAD4, a transforming growth factor- $\beta$ pathway regulator, and suppresses its translocation into the nucleus, where it functions as an oncosuppressor. Apart from suppressing oncosuppressors, ORF1p can also activate oncogenes. In a breast cancer cell line, ${ }^{76}$ ORF1p promoted cell proliferation and invasion via enhancing ETS-1 transcriptional activity and thus increasing the expression of downstream oncogenes that regulate cancer invasion and metastasis. In a human CRC cell line and xenograft mouse model, ${ }^{77}$ similar results were observed regarding the effects of ORF1p on cancer cell behaviors, ETS-1 transcriptional activity, and downstream gene expression. These examples demonstrate the potential oncogenic implications of ORF1p overexpression and indicate its possible use as a biomarker and as a future target for potential therapeutic agents.

\section{ORF2p}

ORF2p possesses endonuclease and RT activities, and thus is indispensable for L1 retrotransposition. In a transgenic mouse model of breast cancer, ${ }^{33}$ ORF2p RT activity was detectable in the cytoplasm at an early tumor stage (preceding the detectability of conventional biomarkers of breast cancer) and accumulated in the nucleus during tumor progression. Additionally, two independent studies suggested that treatment with abacavir or efavirenz, two reverse-transcription inhibitors used in anti-HIV therapy, has anticancer effects in prostate ${ }^{78}$ and breast cancer cells, ${ }^{79}$ respectively. Moreover, treatment with efavirenz in breast cancer cells led to reprogramming of transcriptional profile such as downregulation of genes regulating cell proliferation, migration, and invasion. ${ }^{79}$ Currently, efavirenz has been evaluated in clinical trial as a therapeutic agent for metastatic prostate cancer. $^{80}$ Although in general the trial failed to observe a statistically significant effect on the progression of prostate cancer, it suggested potential benefit in a small subgroup of patients with optimal plasma efavirenz concentration. ${ }^{80}$ Both the presence of ORF2p RT in cancer cells and the possible anticancer effects of its inhibitors suggest that it may play an oncogenic role. As stated, many regulatory RNAs derive from L1 transcripts. L1 transcripts can either form dsRNAs, which are processed into miRNAs or siRNAs, or be reverse-transcribed into cDNA and undergo retrotransposition. Recent evidence ${ }^{69}$ showed that ORF2p RT governs the balance between dsRNA formation and retrotransposition. In cancer, the balance is biased toward retrotransposition, potentially impairing regulatory RNA formation and meanwhile increasing retrotransposition-induced mutagenesis. Therefore, RT inhibition may represent a promising area for the development of anticancer strategies in the future.

\section{PERSPECTIVES: IMPLICATIONS OF L1 ACTIVITY AND EXPRESSION IN CANCER DIAGNOSES AND POTENTIAL THERAPIES}

Although mounting evidence supports that global hypomethylation involving L1 regions is an important cause of L1 reactivation in cancer, ${ }^{37,38,59}$ upstream regulators of this activation remain elusive. Previous studies have suggested some of the upstream regulators, such as oxidative stress, ${ }^{81,82}$ interleukin- $6,{ }^{83}$ $\operatorname{Rad} 21,{ }^{83}$ and $\mathrm{p} 53$ pathway. ${ }^{31,84}$

Oxidative stress can promote L1 hypomethylation and L1 expression in cancer cell lines, along with disrupted expression of genes involved in DNA repair. ${ }^{81,82}$ Additionally, some precancerous conditions, such as chronic inflammation, can stimulate oxidative stress. ${ }^{85,86}$ Therefore, oxidative stress-induced L1 activation might represent one of the mechanisms linking chronic inflammation and tumorigenesis, which deserves further investigation. Conversely, hypoxia and inflammation in the malignant microenvironment can induce oxidative stress that may stimulate L1 expression. In other words, oxidative stress-induced L1 expression might lie in both upstream and downstream of malignant transformation. Interleukin-6 is an important participant and mediator of chronic inflammation. One study ${ }^{83}$ in a cell line of oral squamous cell carcinoma revealed that interleukin- 6 can induce L1 hypomethylation, suggesting that chronic inflammation preceding and during tumorigenicity may provide a permissive or supporting environment for L1 reactivation. $\operatorname{Rad} 21$ is a member of the cohesin family, which is activated by the Wnt/ $\beta$-catenin pathway. In human CRC cell lines, ${ }^{87} \mathrm{~L} 1$ was found to be activated 
by $\operatorname{Rad} 21$, leading to speculation that L1 activation might be linked to the oncogenic Wnt/ $\beta$-catenin pathway. Associations between p53 deficiency and L1 expression or hypomethylation were observed in multiple cancers. ${ }^{31,84}$ The mechanisms underlying these associations are still elusive. One possible explanation is that L1-induced genomic lesions trigger p53-mediated responses to arrest the growth or to induce the apoptosis of L1-expressing cells, whereas p53 deficiency increases the viability of L1-expressing cells by circumventing these responses.

Although an increasing body of work is being generated on L1 expression and increased retrotransposition, our current knowledge on the regulations of L1 hypomethylation and activation are still in its infancy. We do not know which among the $\mathrm{L} 1$ retrotransposition events that occurred preceding and during tumorigenesis are causes and which are consequences of malignant transformation. We are not clear about to what extent those cancer-related L1s affect tumorigenesis processes and how exactly they are activated. Addressing these questions can stimulate exploration of methods to specifically inhibit the activity of driver L1s, whose mutagenesis can contribute to changes affecting cancer initiation and progression, or conversely, methods to activate L1s to induce genomic lesions to kill cancer cells.

Although the implications of L1 as a therapeutic target are poorly understood, L1 expression may serve as a promising biomarker for cancer diagnosis, subtyping, and reclassification. A recent study, for example, observed that the methylation levels of L1 declined in a stepwise manner in normal endometrium, endometriotic ovarian cysts, ovarian endometrioid adenocarcinoma, and ovarian clear cell carcinoma, thus holding diagnostic potentials. ${ }^{88}$ Cancer subtyping and reclassification can provide custom-designed diagnosis and guide individualized therapy. Hoadley et al., ${ }^{89}$ for example, reclassified 3,527 cancer samples (across 12 tissue-of-origin cancer types) into 11 new types based on six kinds of multi-omic data (including exon, copy number variation, DNA methylation, miRNA, mRNA, and protein) and found that these new types are capable of providing independent prognostic power. As stated, L1 activity profiles differ among and within cancer types $^{32,35}$ and fluctuate during cancer evolution. ${ }^{32-34,37}$ Although these fluctuations might be to some extent due to variation in the cohort of polymorphic L1s present within a given genome, the activity profiles of L1 still warrant further investigation for potential use as a biomarker. Moreover, L1 components, such as L1-derived transcripts ORF1p and ORF2p, all show some extent of cancer-specific expression profiles. ${ }^{31,74}$ Further research is required to clarify L1 activity profiles preceding and during cancer initiation and evolution, as well as in response to cancer therapy.

\section{CONCLUDING REMARKS}

In conclusion, $\mathrm{L} 1$ retrotransposition, a process active during embryogenesis but epigenetically repressed in normal somatic tissues, is reactivated in cancer, causing genomic lesions and epigenetic alterations. L1 expression correlates with tumorigenesis in some cancers and may contribute to the process of transformation in a minority of cases. Moreover, previous studies $^{32,33,35,37,38}$ in a small number of cancer types have shown that L1 activity and expression differ among and within cancer types and may fluctuate during cancer evolution, suggesting its potential as a cancer biomarker. Future study is still required to illustrate why L1 activity and expression are deregulated in cancer and how they contribute to tumorigenesis.

\section{DISCLAIMER}

Owing to space limitations, some references have regrettably been omitted, particularly those reporting findings for which no or little controversy exists.

\section{DISCLOSURE}

The authors declare no conflict of interest.

\section{REFERENCES}

1. Lander ES, Linton LM, Birren B, et al.; International Human Genome Sequencing Consortium. Initial sequencing and analysis of the human genome. Nature 2001;409:860-921.

2. Brouha B, Schustak J, Badge RM, et al. Hot L1s account for the bulk of retrotransposition in the human population. Proc Natl Acad Sci USA 2003; 100:5280-5285.

3. Miki Y, Nishisho I, Horii A, et al. Disruption of the APC gene by a retrotransposal insertion of L1 sequence in a colon cancer. Cancer Res 1992;52:643-645.

4. Kaer K, Speek M. Retroelements in human disease. Gene 2013;518:231-241.

5. Chen JM, Stenson PD, Cooper DN, Férec C. A systematic analysis of LINE-1 endonuclease-dependent retrotranspositional events causing human genetic disease. Hum Genet 2005;117:411-427.

6. Dombroski BA, Mathias SL, Nanthakumar E, Scott AF, Kazazian HH Jr. Isolation of an active human transposable element. Science 1991;254:1805-1808.

7. Swergold GD. Identification, characterization, and cell specificity of a human LINE-1 promoter. Mol Cell Biol 1990;10:6718-6729.

8. Speek M. Antisense promoter of human L1 retrotransposon drives transcription of adjacent cellular genes. Mol Cell Biol 2001;21:1973-1985.

9. Cruickshanks HA, Vafadar-Isfahani N, Dunican DS, et al. Expression of a large LINE-1-driven antisense RNA is linked to epigenetic silencing of the metastasis suppressor gene TFPI-2 in cancer. Nucleic Acids Res 2013;41:6857-6869.

10. Wei W, Gilbert N, Ooi SL, et al. Human L1 retrotransposition: cis preference versus trans complementation. Mol Cell Biol 2001;21:1429-1439.

11. Kulpa DA, Moran JV. Cis-preferential LINE-1 reverse transcriptase activity in ribonucleoprotein particles. Nat Struct Mol Biol 2006;13:655-660.

12. Luan DD, Korman MH, Jakubczak JL, Eickbush TH. Reverse transcription of R2Bm RNA is primed by a nick at the chromosomal target site: a mechanism for non-LTR retrotransposition. Cell 1993;72:595-605.

13. Morrish TA, Gilbert N, Myers JS, et al. DNA repair mediated by endonucleaseindependent LINE-1 retrotransposition. Nat Genet 2002;31:159-165.

14. Ade C, Roy-Engel AM, Deininger PL. Alu elements: an intrinsic source of human genome instability. Curr Opin Virol 2013;3:639-645.

15. Babatz TD, Burns KH. Functional impact of the human mobilome. Curr Opin Genet Dev 2013;23:264-270.

16. Raiz J, Damert A, Chira $S$, et al. The non-autonomous retrotransposon SVA is trans-mobilized by the human LINE-1 protein machinery. Nucleic Acids Res 2012:40:1666-1683.

17. Cooke SL, Shlien A, Marshall J, et al.; ICGC Breast Cancer Group. Processed pseudogenes acquired somatically during cancer development. Nat Commun 2014:5:3644.

18. Soifer HS, Zaragoza A, Peyvan M, Behlke MA, Rossi JJ. A potential role for RNA interference in controlling the activity of the human LINE-1 retrotransposon. Nucleic Acids Res 2005;33:846-856.

19. Yang N, Kazazian HH Jr. L1 retrotransposition is suppressed by endogenously encoded small interfering RNAs in human cultured cells. Nat Struct Mol Biol 2006:13:763-771. 
20. Sienski G, Dönertas D, Brennecke J. Transcriptional silencing of transposons by Piwi and maelstrom and its impact on chromatin state and gene expression. Cell 2012;151:964-980.

21. De Fazio S, Bartonicek N, Di Giacomo M, et al. The endonuclease activity of Mili fuels piRNA amplification that silences LINE1 elements. Nature 2011;480: 259-263.

22. Sigurdsson MI, Smith AV, Bjornsson HT, Jonsson JJ. The distribution of a germline methylation marker suggests a regional mechanism of LINE-1 silencing by the piRNA-PIWI system. BMC Genet 2012;13:31

23. Pezic $D$, Manakov SA, Sachidanandam R, Aravin AA. piRNA pathway targets active LINE1 elements to establish the repressive H3K9me3 mark in germ cells. Genes Dev 2014;28:1410-1428.

24. Liu C, Zhang $X$, Huang F, et al. APOBEC3G inhibits microRNA-mediated repression of translation by interfering with the interaction between Argonaute-2 and MOV10. J Biol Chem 2012;287:29373-29383.

25. Kinomoto M, Kanno T, Shimura M, et al. All APOBEC3 family proteins differentially inhibit LINE-1 retrotransposition. Nucleic Acids Res 2007;35: 2955-2964.

26. Koito A, Ikeda T. Intrinsic restriction activity by AID/APOBEC family of enzymes against the mobility of retroelements. Mob Genet Elements 2011;1:197-202.

27. Richardson SR, Narvaiza I, Planegger RA, Weitzman MD, Moran JV. APOBEC3A deaminates transiently exposed single-strand DNA during LINE-1 retrotransposition. Elife 2014;3:e02008.

28. Horn AV, Klawitter S, Held U, et al. Human LINE-1 restriction by APOBEC $3 \mathrm{C}$ is deaminase independent and mediated by an ORF1p interaction that affects LINE reverse transcriptase activity. Nucleic Acids Res 2014;42:396-416.

29. Brouha B, Meischl C, Ostertag E, et al. Evidence consistent with human L1 retrotransposition in maternal meiosis I. Am J Hum Genet 2002;71:327-336.

30. van den Hurk JA, Meij IC, Seleme MC, et al. L1 retrotransposition can occur early in human embryonic development. Hum Mol Genet 2007;16:1587-1592.

31. Rodić N, Sharma R, Sharma R, et al. Long interspersed element-1 protein expression is a hallmark of many human cancers. Am J Pathol 2014;184: 1280-1286.

32. Ashktorab $H$, Daremipouran $M$, Goel $A$, et al. DNA methylome profiling identifies novel methylated genes in African American patients with colorectal neoplasia. Epigenetics 2014;9:503-512.

33. Gualtieri A, Andreola F, Sciamanna I, Sinibaldi-Vallebona P, Serafino A, Spadafora C. Increased expression and copy number amplification of LINE1 and SINE B1 retrotransposable elements in murine mammary carcinoma progression. Oncotarget 2013:4:1882-1893.

34. Iwagami S, Baba Y, Watanabe M, et al. LINE-1 hypomethylation is associated with a poor prognosis among patients with curatively resected esophageal squamous cell carcinoma. Ann Surg 2013;257:449-455.

35. Lee E, Iskow R, Yang L, et al.; Cancer Genome Atlas Research Network. Landscape of somatic retrotransposition in human cancers. Science 2012;337:967-971.

36. Morse B, Rotherg PG, South VJ, Spandorfer JM, Astrin SM. Insertional mutagenesis of the myc locus by a LINE-1 sequence in a human breast carcinoma. Nature 1988;333:87-90.

37. Tubio JM, Li Y, Ju YS, et al.; ICGC Breast Cancer Group; ICGC Bone Cancer Group; ICGC Prostate Cancer Group. Mobile DNA in cancer. Extensive transduction of nonrepetitive DNA mediated by L1 retrotransposition in cancer genomes. Science 2014;345:1251343.

38. Hur K, Cejas P, Feliu J, et al. Hypomethylation of long interspersed nuclear element-1 (LINE-1) leads to activation of proto-oncogenes in human colorectal cancer metastasis. Gut 2014;63:635-646.

39. Beroukhim R, Getz G, Nghiemphu L, et al. Assessing the significance of chromosomal aberrations in cancer: methodology and application to glioma. Proc Natl Acad Sci USA 2007;104:20007-20012.

40. Chappell G, Kutanzi K, Uehara T, et al. Genetic and epigenetic changes in fibrosis-associated hepatocarcinogenesis in mice. Int J Cancer 2014;134: 2778-2788.

41. Deroo LA, Bolick SC, Xu Z, et al. Global DNA methylation and one-carbon metabolism gene polymorphisms and the risk of breast cancer in the Sister Study. Carcinogenesis 2014;35:333-338.

42. Ogino S, Nishihara R, Lochhead P, et al. Prospective study of family history and colorectal cancer risk by tumor LINE-1 methylation level. J Natl Cancer Inst 2013;105:130-140.

43. Kamiyama H, Suzuki $\mathrm{K}$, Maeda $\mathrm{T}$, et al. DNA demethylation in normal colon tissue predicts predisposition to multiple cancers. Oncogene 2012;31: 5029-5037.
44. Shuangshoti S, Hourpai N, Pumsuk U, Mutirangura A. Line-1 hypomethylation in multistage carcinogenesis of the uterine cervix. Asian Pac J Cancer Prev 2007;8:307-309.

45. Inamura K, Yamauchi M, Nishihara R, et al. Tumor LINE-1 methylation level and microsatellite instability in relation to colorectal cancer prognosis. J Nat/ Cancer Inst 2014;106.

46. Zhu C, Utsunomiya T, Ikemoto T, et al. Hypomethylation of long interspersed nuclear element-1 (LINE-1) is associated with poor prognosis via activation of c-MET in hepatocellular carcinoma. Ann Surg Oncol 2014;21(suppl 4): S729-S735.

47. Shigaki H, Baba $Y$, Watanabe $M$, et al. LINE-1 hypomethylation in gastric cancer, detected by bisulfite pyrosequencing, is associated with poor prognosis. Gastric Cancer 2013;16:480-487.

48. Li J, Huang Q, Zeng F, et al. The prognostic value of global DNA hypomethylation in cancer: a meta-analysis. PLoS One 2014;9:e106290.

49. Neale RE, Clark PJ, Fawcett J, et al. Association between hypermethylation of DNA repetitive elements in white blood cell DNA and pancreatic cancer. Cancer Epidemiol 2014;38:576-582.

50. Akers SN, Moysich K, Zhang W, et al. LINE1 and Alu repetitive element DNA methylation in tumors and white blood cells from epithelial ovarian cancer patients. Gynecol Oncol 2014;132:462-467.

51. Walters RJ, Williamson EJ, English DR, et al. Association between hypermethylation of DNA repetitive elements in white blood cell DNA and earlyonset colorectal cancer. Epigenetics 2013;8:748-755.

52. Hoshimoto S, Kuo CT, Chong KK, et al. AIM1 and LINE-1 epigenetic aberrations in tumor and serum relate to melanoma progression and disease outcome. $J$ Invest Dermato/ 2012;132:1689-1697.

53. Ramzy II, Omran DA, Hamad O, Shaker O, Abboud A. Evaluation of serum LINE1 hypomethylation as a prognostic marker for hepatocellular carcinoma. Arab J Gastroentero/ 2011;12:139-142.

54. Wu HC, Delgado-Cruzata L, Flom JD, et al. Repetitive element DNA methylation levels in white blood cell DNA from sisters discordant for breast cancer from the New York site of the Breast Cancer Family Registry. Carcinogenesis 2012;33:1946-1952

55. Tahara T, Maegawa S, Chung W, et al. Examination of whole blood DNA methylation as a potential risk marker for gastric cancer. Cancer Prev Res (Phila) 2013:6:1093-1100.

56. Gao Y, Baccarelli A, Shu XO, et al. Blood leukocyte Alu and LINE-1 methylation and gastric cancer risk in the Shanghai Women's Health Study. Br J Cancer 2012;106:585-591.

57. Kaer K, Branovets J, Hallikma A, Nigumann P, Speek M. Intronic L1 retrotransposons and nested genes cause transcriptional interference by inducing intron retention, exonization and cryptic polyadenylation. PLoS One 2011:6:e26099.

58. Wanichnopparat W, Suwanwongse K, Pin-On P, Aporntewan C, Mutirangura A. Genes associated with the cis-regulatory functions of intragenic LINE-1 elements. BMC Genomics 2013;14:205.

59. Shukla R, Upton KR, Muñoz-Lopez M, et al. Endogenous retrotransposition activates oncogenic pathways in hepatocellular carcinoma. Cell 2013;153: 101-111.

60. Han K, Sen SK, Wang J, et al. Genomic rearrangements by LINE-1 insertionmediated deletion in the human and chimpanzee lineages. Nucleic Acids Res 2005:33:4040-4052

61. Gilbert N, Lutz S, Morrish TA, Moran JV. Multiple fates of L1 retrotransposition intermediates in cultured human cells. Mol Cell Biol 2005;25: 7780-7795.

62. Xiao-Jie L, Ai-Mei G, Li-Juan J, Jiang X. Pseudogene in cancer: real functions and promising signature. J Med Genet 2015;52:17-24.

63. Moran JV, DeBerardinis RJ, Kazazian HH Jr. Exon shuffling by L1 retrotransposition. Science 1999;283:1530-1534.

64. Chow JC, Ciaudo C, Fazzari MJ, et al. LINE-1 activity in facultative heterochromatin formation during $\mathrm{X}$ chromosome inactivation. Cell 2010;141:956-969.

65. Bojang P Jr, Roberts RA, Anderton MJ, Ramos KS. Reprogramming of the HepG2 genome by long interspersed nuclear element-1. Mol Oncol 2013;7:812-825.

66. Spengler RM, Oakley CK, Davidson BL. Functional microRNAs and target sites are created by lineage-specific transposition. Hum Mol Genet 2014;23: 1783-1793.

67. Kapusta A, Kronenberg Z, Lynch VJ, et al. Transposable elements are major contributors to the origin, diversification, and regulation of vertebrate long noncoding RNAs. PLoS Genet 2013;9:e1003470. 
68. Cheng DL, Xiang YY, Ji LJ, Lu XJ. Competing endogenous RNA interplay in cancer: mechanism, methodology, and perspectives. Tumour Biol 2015; 36:479-488.

69. Sciamanna I, Gualtieri A, Cossetti C, et al. A tumor-promoting mechanism mediated by retrotransposon-encoded reverse transcriptase is active in human transformed cell lines. Oncotarget 2013;4:2271-2287.

70. Lau CC, Sun T, Ching AK, et al. Viral-human chimeric transcript predisposes risk to liver cancer development and progression. Cancer Cell 2014;25:335-349.

71. Han JS, Szak ST, Boeke JD. Transcriptional disruption by the L1 retrotransposon and implications for mammalian transcriptomes. Nature 2004;429:268-274.

72. DiFeo A, Martignetti JA, Narla G. The role of KLF6 and its splice variants in cancer therapy. Drug Resist Updat 2009;12:1-7.

73. Chen J, Weiss WA. Alternative splicing in cancer: implications for biology and therapy. Oncogene 2015;34:1-14.

74. Chen L, Dahlstrom JE, Chandra A, Board P, Rangasamy D. Prognostic value of LINE-1 retrotransposon expression and its subcellular localization in breast cancer. Breast Cancer Res Treat 2012;136:129-142.

75. Zhu Y, Feng F, Yu J, et al. L1-ORF1p, a Smad4 interaction protein, promotes proliferation of HepG2 cells and tumorigenesis in mice. DNA Cell Biol 2013:32:531-540.

76. Yang Q, Feng F, Zhang F, et al. LINE-1 ORF-1p functions as a novel HGF/ETS-1 signaling pathway co-activator and promotes the growth of MDA-MB-231 cell. Cell Signal 2013;25:2652-2660.

77. Li MY, Zhu M, Feng F, et al. Long interspersed nucleotide acid element-1 ORF-1 protein promotes proliferation and invasion of human colorectal cancer LoVo cells through enhancing ETS-1 activity. Genet Mol Res 2014;13:6981-6994.

78. Carlini F, Ridolfi B, Molinari A, et al. The reverse transcription inhibitor abacavir shows anticancer activity in prostate cancer cell lines. PLoS One 2010;5: e14221.

79. Patnala R, Lee SH, Dahlstrom JE, et al. Inhibition of LINE-1 retrotransposonencoded reverse transcriptase modulates the expression of cell differentiation genes in breast cancer cells. Breast Cancer Res Treat 2014;143:239-253.
80. Houédé N, Pulido M, Mourey L, et al. A phase II trial evaluating the efficacy and safety of efavirenz in metastatic castration-resistant prostate cancer. Oncologist 2014;19:1227-1228.

81. Wongpaiboonwattana W, Tosukhowong P, Dissayabutra T, Mutirangura A, Boonla C. Oxidative stress induces hypomethylation of LINE-1 and hypermethylation of the RUNX3 promoter in a bladder cancer cell line. Asian Pac J Cancer Prev 2013;14:3773-3778.

82. Giorgi G, Marcantonio P, Del Re B. LINE-1 retrotransposition in human neuroblastoma cells is affected by oxidative stress. Cell Tissue Res 2011;346:383-391.

83. Gasche JA, Hoffmann J, Boland CR, Goel A. Interleukin-6 promotes tumorigenesis by altering DNA methylation in oral cancer cells. Int J Cancer 2011;129:1053-1063.

84. Morikawa T, Kuchiba A, Liao X, et al. Tumor TP53 expression status, body mass index and prognosis in colorectal cancer. Int J Cancer 2012;131: 1169-1178.

85. Ohnishi S, Ma N, Thanan R, et al. DNA damage in inflammation-related carcinogenesis and cancer stem cells. Oxid Med Cell Longev 2013;2013: 387014.

86. Lonkar P, Dedon PC. Reactive species and DNA damage in chronic inflammation: reconciling chemical mechanisms and biological fates. Int J Cancer 2011;128:1999-2009.

87. Xu H, Yan Y, Deb S, et al. Cohesin Rad21 mediates loss of heterozygosity and is upregulated via Wnt promoting transcriptional dysregulation in gastrointestinal tumors. Cell Rep 2014;9:1781-1797.

88. Senthong A, Kitkumthorn N, Rattanatanyong P, Khemapech N, Triratanachart S, Mutirangura A. Differences in LINE-1 methylation between endometriotic ovarian cyst and endometriosis-associated ovarian cancer. Int J Gynecol Cancer 2014;24:36-42.

89. Hoadley KA, Yau C, Wolf DM, et al.; Cancer Genome Atlas Research Network. Multiplatform analysis of 12 cancer types reveals molecular classification within and across tissues of origin. Cell 2014;158:929-944. 